\title{
Experimental Demonstration of Frequency Autolocking an Optical Cavity Using a Time-Varying Kalman Filter
}

\author{
Dirk Schütte, ${ }^{1}$ S. Z. Sayed Hassen, ${ }^{2,3}$ Kai S. Karvinen, ${ }^{1}$ Toby K. Boyson, ${ }^{2}$ Abhijit G. Kallapur, ${ }^{2}$ \\ Hongbin Song, ${ }^{2,4}$ Ian R. Petersen, ${ }^{2}$ Elanor H. Huntington, ${ }^{2,5}$ and Michèle Heurs ${ }^{1}$ \\ ${ }^{1}$ Max Planck Institute for Gravitational Physics (Albert Einstein Institute), and Institut für \\ Gravitationsphysik, Leibniz Universität Hannover, Callinstraße 38, 30167 Hannover, Germany \\ ${ }^{2}$ School of Engineering and Information Technology, The University of New South Wales, \\ Australian Defence Force Academy, Australian Capital Territory 2600, Australia \\ ${ }^{3}$ Department of Electrical and Electronic Engineering, University of Mauritius, Réduit, Mauritius \\ ${ }^{4}$ School of Humanities and Social Science, The Chinese University of Hong Kong, \\ Shenzhen 518000, China \\ ${ }^{5}$ The Australian National University, College of Engineering \& Computer Science, \\ Australian Capital Territory 0200, Australia \\ (Received 8 May 2015; revised manuscript received 2 October 2015; published 13 January 2016)
}

\begin{abstract}
We propose and demonstrate a new autolocking scheme using a three-mirror ring cavity consisting of a linear quadratic regulator and a time-varying Kalman filter. Our technique does not require a frequency scan to acquire resonance. We utilize the singular perturbation method to simplify our system dynamics and to permit the application of linear control techniques. The error signal combined with the transmitted power is used to estimate the cavity detuning. This estimate is used by a linear time-varying Kalman filter which enables the implementation of an optimal controller. The experimental results validate the controller design, and we demonstrate improved robustness to disturbances and a faster locking time than a traditional proportional-integral controller. More important, the time-varying Kalman filtering approach automatically reacquires lock for large detunings, where the error signal leaves its linear capture range, a feat which linear time-invariant controllers cannot achieve.
\end{abstract}

DOI: 10.1103/PhysRevApplied.5.014005

\section{INTRODUCTION}

Cavity locking is a ubiquitous problem in many fields of research employing single-frequency lasers. For applications in fields such as spectroscopy [1,2], quantum information [3,4], optical imaging [5], and many others, it is necessary to lock the cavity length of an optical resonator to the frequency of a laser (or vice versa) to achieve transmission of the light through the cavity (or a desired frequency noise reduction). One of the most advanced applications that necessitates cavity locking is interferometric gravitational-wave detection [6,7], where numerous controllers are employed to ensure high-dutycycle operation. To maximize the duty cycle, it is essential to ensure rapid relocking of all cavities. Typical schemes used for cavity locking (or, correspondingly, laser frequency locking) include the Pound-Drever-Hall technique [8], Hänsch-Couillaud locking [9], homodyne locking [10], and midfringe locking [11]. The error signal required for cavity locking is inherently nonlinear, rendering lock acquisition nontrivial. In general, when cavity lock is lost, lock reacquisition is achieved by scanning the cavity length (usually with a piezoelectric transducer) until a resonance is found, then interrupting the scan and engaging the controller. This process can be automated to speed up relocking (termed autolocking [12]).
In this paper, we demonstrate a new autolocking scheme based on modern control techniques using a linear timevarying Kalman filter combined with a linear quadratic regulator (LQR). This scheme was proposed in Ref. [13] and simulations suggested reliable, more robust, and faster frequency-lock acquisition than traditional proportionalintegral (PI) control.

Kalman filtering is an effective modern control technique, which can be employed to estimate the state of a dynamical system using noise-corrupted measurements. For cavity locking, a phase-sensitive measurement and an amplitude measurement are required to quantitatively determine the cavity detuning. In our setup, we utilize the error signal generated by homodyne locking and the transmitted power signal to estimate the detuning, which is used by the Kalman filter to estimate the system state. The successful implementation of a LQR controller and a time-invariant Kalman filter for cavity locking was previously demonstrated in Ref. [14]. However, linear timeinvariant controllers are unable to acquire lock if the system is operating in the nonlinear regime.

For the implementation of our controller, we set up an optical plant, which we describe in Sec. II. In Sec. III, we introduce linear quadratic Gaussian control (LQG). We need to make simplifying assumptions to enable the 
application of linear control and estimation techniques, as outlined in Secs. IV and V. Details regarding the realization of our control scheme are given in Sec. VI. The results are presented in Sec. VII as a comparison with a PI controller, highlighting our controller's improved performance and that our controller does not fail in the nonlinear region. Moreover, our controller design automatically achieves frequency lock from any given operating point without requiring a frequency scan.

\section{THE OPTICAL PLANT}

We set up a three-mirror ring cavity as shown schematically in Fig. 1 to demonstrate operation of the proposed control scheme. The parameters of the optical resonator (given in Table I) are chosen to provide easy and convenient handling of the system to test new locking schemes. For this reason, a cavity with a low finesse of $\mathcal{F} \approx 10$ and a large spectral linewidth of $\Delta \nu \approx 65 \mathrm{MHz}$ (corresponding to a decay rate $\kappa \approx 410 \times 10^{6} \mathrm{rad} / \mathrm{s}$ ) is constructed. This results in a small power buildup within the optical resonator and a suitably large linear region of the error signal. The two measured output signals are $y_{1}$, the phase quadrature of the reflected beam, measured via homodyne detection, and $y_{2}$, the intensity of the transmitted beam measured by a single photodetector; the measured signals are shown in Fig. 6 (see Sec. VII). Complete knowledge of the plant is required for the modern control approach described in Secs. III-V.

First, we describe the dynamics of the optical system using the Heisenberg picture with the following cavity differential equations [15]:

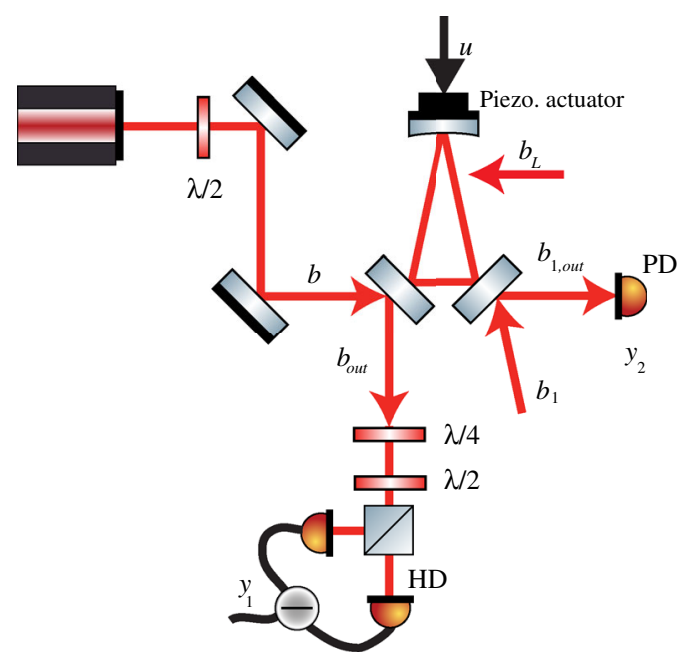

FIG. 1. Schematic of the optical plant including the optical fields $b_{i}$, the control input to the piezoelectric actuator $u$, and the measured outputs $y_{1}$ and $y_{2}$ corresponding to the error signal and the transmitted signal, respectively. HD, $\mathrm{PD}, \lambda / 2$, and $\lambda / 4$ refer to homodyne detector, single photodetector, half-wave, and quarterwave plate, respectively.
TABLE I. Parameters of the optical plant.

\begin{tabular}{lc}
\hline \hline Parameter & Value \\
\hline Wavelength & $1550 \mathrm{~nm}$ \\
Finesse & $\approx 10$ \\
Spectral linewidth & $\approx 65 \mathrm{MHz}$ \\
Waist & $453 \mu \mathrm{m}$ \\
\hline \hline
\end{tabular}

$\dot{a}=-\left(\frac{\kappa}{2}-i \Delta\right) a-\sqrt{\kappa}_{0}\left(\beta+b_{0}\right)-\sqrt{\kappa}_{1} b_{1}-\sqrt{\kappa}_{L} b_{L}$,

$$
\begin{aligned}
b_{\text {out }} & =\sqrt{\kappa}_{0} a+\beta+b_{0}, \\
b_{1, \text { out }} & =\sqrt{\kappa}_{1} a+b_{1},
\end{aligned}
$$

where $a$ is the annihilation operator of the intracavity field and $b$ the annihilation operator of the input field. The cavity input from the laser can be expressed as a boson field $b=\beta+b_{0}$, where the operator $b$ is written as a combination of a complex number $\beta$ (describing the coherent amplitude of the field) and an operator $b_{0}$ (describing the fluctuations of the field). $\kappa$ is the total decay rate of the cavity and is the sum of the individual decay rates $\kappa_{0}, \kappa_{1}$, and $\kappa_{L}$ of the optical fields $b, b_{1}$ (the input field from the rear plane mirror or output coupler), and $b_{L}$ (the optical loss field) coupling to the cavity, respectively,

$$
\kappa=\kappa_{0}+\kappa_{1}+\kappa_{L} .
$$

$\Delta$ is the frequency detuning between the cavity and laser given by

$$
\Delta=f_{c}-f_{L}=q \frac{c}{n L}-f_{L},
$$

where $f_{c}$ is the resonance frequency of the cavity, $f_{L}$ the laser frequency, $n L$ the optical path length of the cavity (with $n$ being the index of refraction and $L$ the physical path length), $c$ the speed of light, and $q$ a large integer indicating the $q$ th longitudinal cavity mode. A good overview of cavity dynamics can be found in Refs. [15,16]. The two measured outputs are given by

$$
\begin{aligned}
y_{1} & =e^{-i \phi} b_{\text {out }}+e^{i \phi} b_{\text {out }}^{\dagger} \\
& =\sqrt{\kappa}_{0}\left(e^{-i \phi} a+e^{i \phi} a^{\dagger}\right)+2 \beta \cos \phi+q_{0}, \\
y_{2} & =b_{1, \text { out }}^{\dagger} b_{1, \text { out }} \\
& =\kappa_{1} a^{\dagger} a+\sqrt{\kappa_{1}}\left(a^{\dagger} b_{1}+b_{1}^{\dagger} a\right)+b_{1}^{\dagger} b_{1},
\end{aligned}
$$

where $q_{0}$ is a Gaussian white noise describing the noise of the input field, and $\phi$ is the homodyne measurement angle. For our polarization-based homodyne locking scheme, $\phi$ 
is determined by the orientation of the quarter-wave- or half-wave-plate combination in the beam path [10]. We rewrite the cavity dynamics in quadrature operator form; the amplitude and phase quadratures $q$ and $p$ are then defined by

$$
q=a+a^{\dagger}, \quad p=i\left(a^{\dagger}-a\right) .
$$

In the following section, we use the quadrature operator form to convert the cavity dynamics into a state-space model which is used to design the controller which consists of a time-varying Kalman filter and a LQR controller.

\section{LINEAR QUADRATIC GAUSSIAN CONTROL}

A systematic modern control approach is extremely beneficial when the complexity of the system under consideration grows beyond the scale where heuristic techniques are viable. In Ref. [14], it was demonstrated that the application of modern control techniques can lead to a cavity frequency lock with improved bandwidth and higher stability margins than a traditional PI controller. In this section, we give an overview of LQG control, and in the following sections, we show how it can be applied to lock the resonance frequency of an optical cavity to the laser frequency. To design a discrete-time LQG controller, a linear discrete-time state-space model of the plant is required; a practical model should also include sensor and actuator dynamics. A time-invariant state-space model without direct feedthrough from the input to the output $(D=0)$ can be expressed in the following form:

$$
\begin{gathered}
x_{k+1}=A x_{k}+B u_{k}+w_{k}, \\
y_{k}=C x_{k}+v_{k},
\end{gathered}
$$

where $x_{k} \in \mathbb{R}^{n}$ is the state vector, $u_{k} \in \mathbb{R}^{m}$ is the input vector, and $y_{k} \in \mathbb{R}^{p}$ is the output vector at time instant $k$. The matrix $A \in \mathbb{R}^{n \times n}$ is called the system or state matrix, and it represents the system dynamics. The input matrix $B \in \mathbb{R}^{n \times m}$ describes how the inputs affect the time evolution of the system, and the output matrix $C \in \mathbb{R}^{p \times n}$ describes how the measured outputs depend on the system states. The process noise $w_{k} \in \mathbb{R}^{m}$ and measurement noise $v_{k} \in \mathbb{R}^{p}$ are both assumed to be white Gaussian noises. A LQG controller consists of the combination of a LQR and a linear quadratic estimator. The LQR problem is an optimal control problem where we seek to optimize the performance of the closed-loop system with respect to a quadratic cost function. A typical infinite-horizon discrete-time cost function $\mathcal{J}$ may take the form

$$
\mathcal{J}=\sum_{k=0}^{\infty} x_{k}^{T} Q x_{k}+u_{k}^{T} R u_{k},
$$

where $Q \in \mathbb{R}^{n \times n}$ and $R \in \mathbb{R}^{m \times m}$ are design parameters. The term $x_{k}^{T} Q x_{k}$ regulates the speed of convergence of the state variables $x_{k}$, and the term $u_{k}^{T} R u_{k}$ sets constraints for the applied control energy. The optimal control law is a static state feedback law of the form

$$
u_{k}=-F x_{k},
$$

where the constant feedback gain $F$ is calculated by

$$
F=\left(B^{T} S B+R\right)^{-1} B^{T} S A,
$$

where $S$ satisfies the algebraic Riccati equation

$$
0=A^{T}\left[S-S B\left(B^{T} S B+R\right)^{-1} B S\right] A-S+Q .
$$

There are several limitations of static state feedback control. First, feedforward control is required to eliminate the steady-state tracking error, and, therefore, perfect knowledge of the plant model is required. Second, with state feedback, the states cannot converge in the presence of constant input or output disturbances. To regulate the detuning $\Delta$ to 0 in the presence of unmodeled external disturbances, such as $1 / f$ laser phase noise, integral control must be included. Integral action can be built into the LQR problem by adding another system state $q_{k+1}=q_{k}+y_{k}$, which integrates the system output. The augmented statespace model then becomes

$$
\begin{aligned}
{\left[\begin{array}{l}
x_{k+1} \\
q_{k+1}
\end{array}\right] } & =\left[\begin{array}{ll}
A & 0 \\
C & I
\end{array}\right]\left[\begin{array}{l}
x_{k} \\
q_{k}
\end{array}\right]+\left[\begin{array}{l}
B \\
0
\end{array}\right] \tilde{u}_{k}+\tilde{w}_{k}, \\
\tilde{y}_{k} & =\left[\begin{array}{ll}
C & 0
\end{array}\right]\left[\begin{array}{l}
x_{k} \\
q_{k}
\end{array}\right]+\tilde{v}_{k} .
\end{aligned}
$$

The LQR control design can be performed on the augmented system $\{\tilde{A}, \tilde{B}, \tilde{C}\}$ where

$$
\begin{array}{ll}
\tilde{A}=\left[\begin{array}{ll}
A & 0 \\
C & I
\end{array}\right], & \tilde{B}=\left[\begin{array}{l}
B \\
0
\end{array}\right], \\
\tilde{C}=\left[\begin{array}{ll}
C & 0
\end{array}\right], & \tilde{x}_{k}=\left[\begin{array}{l}
x_{k} \\
q_{k}
\end{array}\right] .
\end{array}
$$

The resulting controller will be robust to errors in plant modeling, will be able to reject constant disturbances, and we will be able to drive the detuning to 0 .

As shown in Fig. 2(a), the LQR controller requires complete knowledge of the system states; often this knowledge is not accessible and must, therefore, be estimated. The separation principle in control theory states that the design of an optimal feedback controller for a stochastic system can be achieved by designing an optimal estimator and feeding the optimal state estimate into the deterministic optimal feedback controller as shown in Fig. 2(b). 

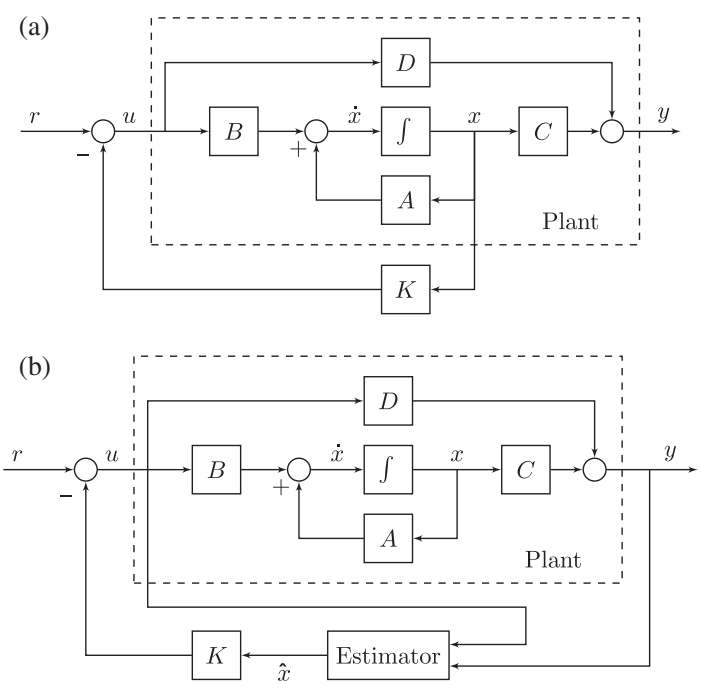

FIG. 2. (a) Linear quadratic regulator with static feedback gain $K$ which is determined by solving the infinite-horizon $\mathrm{LQR}$ problem. The LQR controller requires direct access to the state. (b) LQG controller with feedback of the estimated state $\hat{x}$. Integral action is included when the augmented system is utilized.

The linear quadratic estimation problem is mathematically equivalent to the $\mathrm{LQR}$ problem, where the weighting factors in the cost function reflect the process and measurement noise statistics. When the process and measurement noises are white Gaussian, the optimal estimator is the Kalman filter. The solution of the algebraic Riccati equation determines the steady-state Kalman filter gain. In the time-varying case, the covariance matrix evolves in accordance with the dynamic Riccati equation, thus, causing the Kalman filter gain to vary with time.

For our case, we need to formulate the cavity dynamics in state-space form:

$$
\begin{aligned}
{\left[\begin{array}{c}
\dot{q} \\
\dot{p}
\end{array}\right]=} & {\left[\begin{array}{cc}
-\frac{\kappa}{2} & -\Delta \\
\Delta & -\frac{\kappa}{2}
\end{array}\right]\left[\begin{array}{l}
q \\
p
\end{array}\right]-\left[\begin{array}{c}
2 \beta \sqrt{\kappa_{0}} \\
0
\end{array}\right] } \\
& -\sqrt{\kappa_{0}}\left[\begin{array}{cc}
\cos \phi & \sin \phi \\
-\sin \phi & \cos \phi
\end{array}\right]\left[\begin{array}{l}
q_{0} \\
p_{0}
\end{array}\right] \\
& -\sqrt{\kappa_{1}}\left[\begin{array}{ll}
1 & 0 \\
0 & 1
\end{array}\right]\left[\begin{array}{c}
q_{1} \\
p_{1}
\end{array}\right]-\sqrt{\kappa_{L}}\left[\begin{array}{ll}
1 & 0 \\
0 & 1
\end{array}\right]\left[\begin{array}{c}
q_{L} \\
p_{L}
\end{array}\right], \\
y_{1}= & k_{2}{\sqrt{\kappa_{0}}[\cos \phi}^{\sin \phi]}\left[\begin{array}{c}
q \\
p
\end{array}\right] \\
& +k_{2}\left[\begin{array}{ll}
1 & 0
\end{array}\right]\left[\begin{array}{l}
q_{0} \\
p_{0}
\end{array}\right]+2 k_{2} \beta \cos \phi+\tilde{v}_{1}, \\
y_{2}= & \tilde{k}_{2}\left(\frac{\kappa_{1}}{4}\left(p^{2}+q^{2}\right)+\frac{\sqrt{\kappa_{1}}}{2}\left[\begin{array}{ll}
q & p
\end{array}\right]\left[\begin{array}{l}
q_{1} \\
p_{1}
\end{array}\right]\right)+\tilde{v}_{2},
\end{aligned}
$$

where $\tilde{v}_{1}$ and $\tilde{v}_{2}$ are sensor noises related to the outputs of the system, and $k_{2}, \tilde{k}_{2}$ represent the transimpedance gains of the photodetectors.
Unfortunately, the analytically derived state-space model does not completely describe the physical system; the true physical dynamics are more complex due to the dynamics of the piezoelectric actuator attached to one of the end mirrors to control the cavity length. Hence, we measure the cavity transfer function by injecting a swept sine into the controlled input $u$ and measuring the error signal $y_{1}$ generated via homodyne detection. In the linear range, it is assumed that $\Delta \propto y_{1}$, and, therefore, we identify a model of the following form:

$$
\begin{aligned}
x_{k+1} & =\bar{A} x_{k}+\bar{B} u_{k}, \\
\Delta_{k} & =\bar{C} x_{k} .
\end{aligned}
$$

By utilizing subspace system identification [17], we are able to construct a state-space model of the system, which includes the dynamics of the piezoelectric actuator.

Since, in general, the error signal $y_{1}$ does not vary linearly with respect to the detuning, which also affects the system matrix of the linear state-space model, the control problem is nonlinear. We address this problem by simplifying the system via the singular perturbation method [18] and then implementing a time-varying Kalman filter as described in the following sections.

\section{SINGULAR PERTURBATION METHOD}

To develop a controller that automatically achieves lock from any given operating point, we need to construct an appropriate control law. The control law covers not just the linear, but also the nonlinear region of the generated error signal. We use the singular perturbation method [18] to simplify our system and ensure that a linear control approach can still be applied. The singular perturbation method decomposes the optical cavity into two subsystems which differ by their time constants. This decomposition is called a separation of time scales. In this case, the dynamics of the optical cavity are fast with respect to the mechanical assembly. The slow block, whose output is the detuning, is followed by a fast block, which can be modeled as a static sensor nonlinearity applied to the output. This approach enables the use of linear control techniques. To determine the behavior of the static nonlinearity, we set $\dot{q}=\dot{p}=0$ to obtain

$$
\left[\begin{array}{l}
q \\
p
\end{array}\right]=\frac{-1}{\left(\frac{\kappa}{2}\right)^{2}+\Delta^{2}}\left[\begin{array}{c}
\kappa \beta \sqrt{\kappa_{0}} \\
2 \beta \sqrt{\kappa_{0}} \Delta
\end{array}\right]
$$

For the case when $\phi=\pi / 2$, the outputs $y_{1}$ and $y_{2}$ can be expressed as

$$
\begin{aligned}
y_{1} & =k_{2} \sqrt{\kappa_{0}} p+2 k_{2} \beta \cos \phi+v_{1} \\
& =-\frac{2 k_{2} \beta \kappa_{0} \Delta}{\left(\frac{\kappa}{2}\right)^{2}+\Delta^{2}}+v_{1} \\
& =f_{1}(\Delta)+v_{1},
\end{aligned}
$$




$$
\begin{aligned}
y_{2} & =\frac{\tilde{k}_{2} \kappa_{1}\left(p^{2}+q^{2}\right)}{2}+v_{2}=\frac{1}{2} \frac{\tilde{k}_{2} \kappa_{1} \kappa_{0} \beta^{2}}{\left(\frac{\kappa}{2}\right)^{2}+\Delta^{2}}+v_{2} \\
& =f_{2}(\Delta)+v_{2} .
\end{aligned}
$$

If the optical cavity is perturbed strongly and leaves the linear region of operation, we cannot unambiguously determine the detuning if $y_{1}$ is the only measurement, as shown in Fig. 6. Therefore, we complement $y_{1}$ with $y_{2}$ to correctly ascertain the detuning. Even when both $y_{1}$ and $y_{2}$ are used, both signals tend to zero for very large detuning and become very sensitive to noise. Because of this, we set up a bounded-noise model estimating the actual state of our system via noise-corrupted measurements. In our case, the measurement noise is unknown but bounded. To fulfill this constraint at each time instant, the measurement noises $v_{1, k}$ and $v_{2, k}$ need to satisfy the inequalities

$$
v_{1, k}^{2} \leq \mu_{1}^{2}, \quad v_{2, k}^{2} \leq \mu_{2}^{2},
$$

where $\mu_{1}$ and $\mu_{2}$ are fixed constants denoting the upper bound for the magnitude of the noise. We then consider the problem of characterizing the set of all possible $\Delta_{k}$ compatible with the given observations $y_{1, k}$ and $y_{2, k}$ to find the set $\mathcal{S}_{k}$ that fulfills the inequalities in Eq. (24),

$$
\begin{aligned}
\mathcal{S}_{k}= & \left\{\Delta_{k} \in \mathbb{R}:\left[y_{1, k}-f_{1}\left(\Delta_{k}\right)\right]^{2} \leq \mu_{1}^{2} \quad\right. \text { and } \\
& {\left.\left[y_{2, k}-f_{2}\left(\Delta_{k}\right)\right]^{2} \leq \mu_{2}^{2}\right\} . }
\end{aligned}
$$

Using Eqs. (22)-(24), it is straightforward to verify that $\mathcal{S}_{k}$ corresponds to the set of $\Delta_{k} \in \mathbb{R}$ satisfying the inequalities

$$
\begin{aligned}
& \left(16 \Delta_{k}^{4}+\kappa^{4}\right)\left(y_{1, k}^{2}-\mu_{1}^{2}\right)+64 \Delta_{k}^{3} k_{2} \beta \kappa_{0} y_{1, k} \\
& \quad+8 \Delta_{k}^{2}\left(8 k_{2}^{2} \beta^{2} \kappa_{0}^{2}+\kappa^{2} y_{1, k}^{2}-\kappa^{2} \mu_{1}^{2}\right) \\
& \quad+16 \Delta_{k} k_{2} \beta \kappa_{0} \kappa^{2} y_{1, k} \leq 0 \\
& \left(16 \Delta_{k}^{4}+\kappa^{4}\right)\left(y_{2, k}^{2}-\mu_{2}^{2}\right) \\
& \quad+8 \Delta_{k}^{2}\left(\kappa^{2} y_{2, k}^{2}-4 k_{3} \kappa_{0} \beta^{2} y_{2, k}-\kappa^{2} \mu_{2}^{2}\right) \\
& \quad-8 \kappa^{2} k_{3} \beta^{2} \kappa_{0} y_{2, k}+16 k_{3}^{2} \beta^{4} \kappa_{0}^{2} \leq 0 .
\end{aligned}
$$

The set $\mathcal{S}_{k}$ is constructed by solving the inequalities (26) and (27) using Laguerre's method [19,20]. If we obtain $m$ roots as a solution, they will define $m+1$ regions of interest:

$$
\begin{aligned}
\mathcal{S}_{k, 1} & =\left\{\Delta_{k} \in \mathbb{R}:-\infty<\Delta_{k}<\Delta_{k, 1}\right\}, \\
\mathcal{S}_{k, 2} & =\left\{\Delta_{k} \in \mathbb{R}: \Delta_{k, 1}<\Delta_{k}<\Delta_{k, 2}\right\}, \\
\vdots & \\
\mathcal{S}_{k, m} & =\left\{\Delta_{k} \in \mathbb{R}: \Delta_{k, m-1}<\Delta_{k}<\Delta_{k, m}\right\}, \\
\mathcal{S}_{k, m+1} & =\left\{\Delta_{k} \in \mathbb{R}: \Delta_{k, m}<\Delta_{k}<\infty\right\} .
\end{aligned}
$$

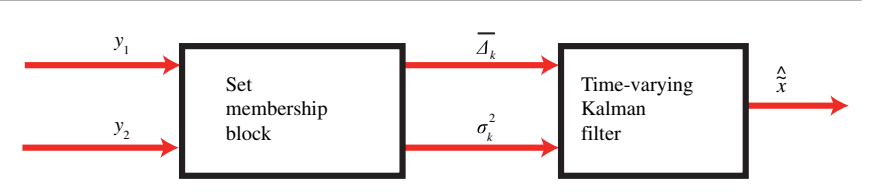

FIG. 3. Block diagram for the calculation of the state estimate $\hat{\tilde{x}}$. $y_{1}, y_{2}$ are the measured signals, $\bar{\Delta}$ is the estimated mean detuning, and $\sigma^{2}$ is the estimated measurement covariance.

The set $\mathcal{S}_{k}$ consists of the subsets $\mathcal{S}_{k, i}$ satisfying Eqs. (26) and (27) at time instant $k$. Let us briefly outline the reasons for this approach. To apply the Kalman filter, we must transform the given measurements $y_{1}$ and $y_{2}$ into the mean detuning $\bar{\Delta}_{k}$ and its variance. Thus, we present a heuristic approach, which attempts to convert the given information into the required quantities. The set $\mathcal{S}_{k}$ can be nonconvex; it is possible for two regions on either side of the resonance peak to satisfy the inequalities when the detuning is large. In this situation, we want to inform the Kalman filter that there is a large uncertainty in this measurement, which is reflected by a large variance. Therefore, when we have a nonconvex solution set we consider $\overline{\mathcal{S}}_{k}$, the convex hull of $\mathcal{S}_{k}$ (i.e., we force the set to become convex by including all possible values between the minimum and maximum values), which makes the set significantly larger with a large variance. On the other hand, when $\mathcal{S}_{k}$ consists of only one subset $\mathcal{S}_{k, i}$, the set is equal to its convex hull $\mathcal{S}_{k}=\overline{\mathcal{S}}_{k}$, and the variance is small, reflecting our increased confidence that this is, in fact, the actual detuning. Taking the convex hull of the solution set is motivated intuitively, and we develop the following equations for the mean value $\bar{\Delta}_{k}$ and the standard deviation $\sigma_{k}$, where we assume a uniform distribution over the convex set $\overline{\mathcal{S}}_{k}$,

$$
\begin{aligned}
\bar{\Delta}_{k} & =\frac{1}{2}\left[\min _{\Delta_{k} \in \overline{\mathcal{S}}_{k}}\left(\Delta_{k}\right)+\max _{\Delta_{k} \in \overline{\mathcal{S}}_{k}}\left(\Delta_{k}\right)\right], \\
\sigma_{k} & =\frac{1}{2 \sqrt{3}}\left[\max _{\Delta_{k} \in \overline{\mathcal{S}}_{k}}\left(\Delta_{k}\right)-\min _{\Delta_{k} \in \overline{\mathcal{S}}_{k}}\left(\Delta_{k}\right)\right] .
\end{aligned}
$$

The quantities $\bar{\Delta}_{k}$ and $\sigma_{k}^{2}$ represent the new measurement for the detuning and the measurement covariance. These quantities are utilized in the time-varying Kalman filter, which is described in the following section. A schematic highlighting this algorithm is shown in Fig. 3. For more algorithm-specific details, refer to the simulations performed in Ref. [13].

\section{TIME-VARYING KALMAN FILTER}

The Kalman filter utilizes the mathematical model of the system and the measurements to estimate inaccessible system states. For an optimal estimate, the process and measurement noises must be known white Gaussian processes. There are two stages to the time-varying Kalman filter: the prediction step and the update step. 


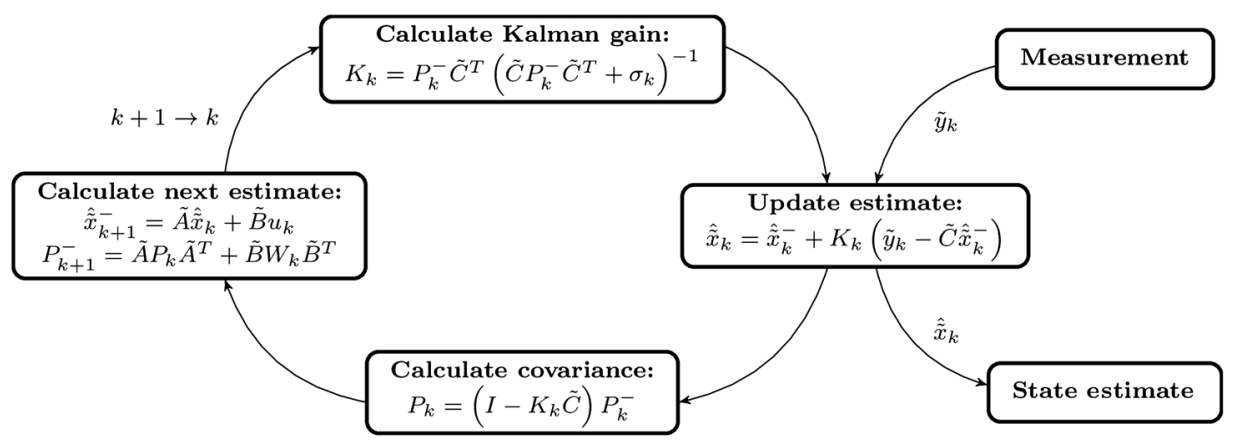

FIG. 4. An overview of the discrete-time Kalman filter.

In the prediction step, the Kalman filter uses the current state estimate $\hat{\tilde{x}}_{k}$ and error covariance $P_{k}$ to predict the state estimate and error covariance at the next time step using the system model,

$$
\begin{aligned}
& \hat{\tilde{x}}_{k+1}^{-}=\tilde{A} \hat{\tilde{x}}_{k}+\tilde{B} \tilde{u}_{k}, \\
& P_{k+1}^{-}=\tilde{A} P_{k} \tilde{A}^{T}+\tilde{B} W \tilde{B}^{T},
\end{aligned}
$$

where $W$ is the covariance of the process noise and is assumed to be diagonal. Here, $\hat{\tilde{x}}_{k}^{-}, P_{k}^{-}$denote the a priori quantities. The a posteriori state estimate and error covariance are given by $\hat{\tilde{x}}_{k}$ and $P_{k}$, respectively.

In the update step, the Kalman filter compares the measured output to the predicted output. The Kalman gain weights the difference between the predicted and measured outputs and optimally adjusts the state estimate. The Kalman gain $K_{k}$ is the optimal weighting factor based on the specified noise statistics, and it determines whether the measurement or the model-generated state estimate is more reliable at each time instant. The Kalman filter is optimal in the sense that it minimizes the variance between the actual and estimated states. The following equations summarize the update step:

$$
\begin{aligned}
& K_{k}=P_{k}^{-} \tilde{C}^{T}\left(\tilde{C} P_{k}^{-} \tilde{C}^{T}+\sigma_{k}\right)^{-1}, \quad P_{0}^{-}=P_{x_{0}}, \\
& \hat{\tilde{x}}_{k}=\hat{\tilde{x}}_{k}^{-}+K_{k}\left(\tilde{y}_{k}-\tilde{C} \hat{\tilde{x}}_{k}^{-}\right), \quad \hat{\tilde{x}}_{0}^{-}=\overline{\tilde{x}}_{0}, \\
& P_{k}=\left(I-K_{k} \tilde{C}\right) P_{k}^{-} .
\end{aligned}
$$

The equations presented here can be found in any standard text on estimation (for more information, see Refs. [21,22]). Figure 4 highlights the recursive nature of the time-varying Kalman filter algorithm. At each time instant $k$, the new measurement $\tilde{y}_{k}=\bar{\Delta}_{k}$ and the measurement covariance $\sigma_{k}$ are determined from Eqs. (28) and (29).

\section{CONTROL IMPLEMENTATION}

The control scheme is implemented on a dSpace DS1103 PPC controller board. The dSpace board consists of eight digital-to-analog-converter and 16 analog-to-digitalconverter channels with a sampling rate of $300 \mathrm{kHz}$.
The board is fully programmable via the MATLAB SIMULINK toolbox and possesses a 16-bit resolution. The computational results related to $\overline{\mathcal{S}}_{k}$ are obtained via a C program; the update rate is $10 \mathrm{kHz}$ to guarantee sufficient bandwidth. The result is a highly robust controller that successfully achieves frequency lock of the optical cavity to the laser from any operating point. While a 12th-order polynomial is solved in the simulation [13], we choose to implement a simpler fourth-order model, which does not include mode splitting. We can safely neglect mode splitting, because the gradients of the error signals corresponding to the nondegenerate $s$ - or $p$-polarized cavity modes differ in sign, as shown in Fig. 6. Hence, it is only possible to lock to one mode at a time; in our case, we choose to lock to the $p$ polarization. A schematic of the closed-loop system is depicted in Fig. 5.

\section{RESULTS}

To validate the proposed controller, we compare its performance to a typical PI controller designed such that the gains of the two controllers and, therefore, their residual

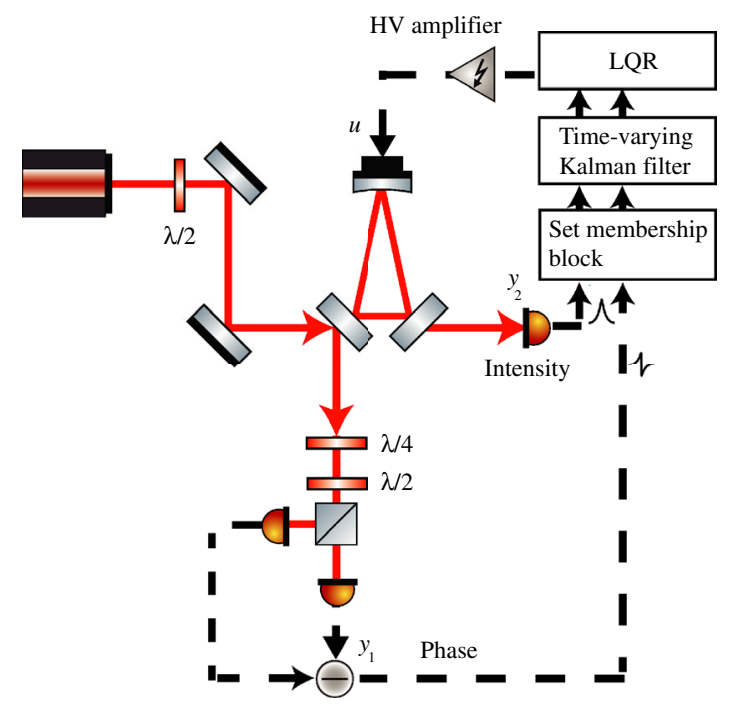

FIG. 5. Schematic of the cavity-locking experiment, including the combination of a time-varying Kalman filter and a linear quadratic regulator. The dashed lines depict electronic links. 

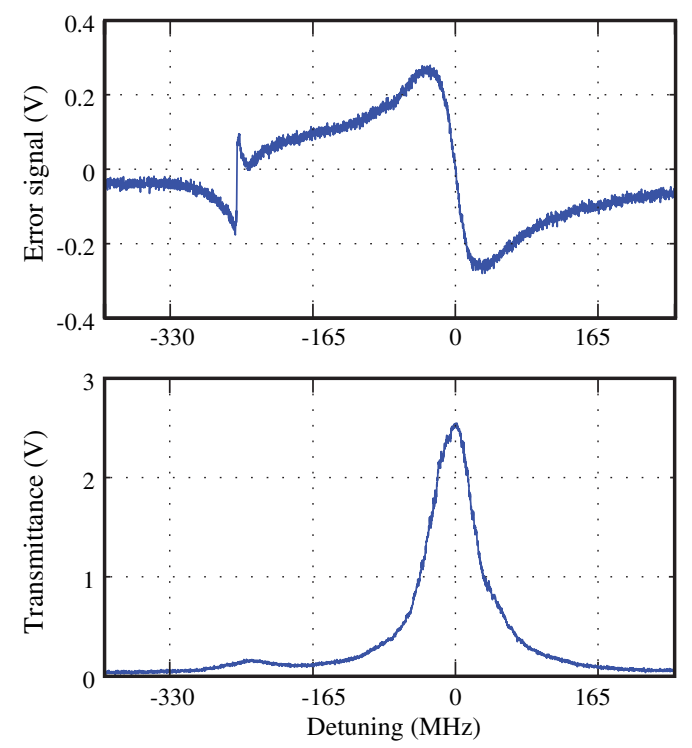

FIG. 6. Frequency response of the optical cavity showing the error signal from the homodyne detector $y_{1}$ (top) and the transmitted signal $y_{2}$ (bottom). The main Airy peak or error signal at $\Delta=0 \mathrm{MHz}$ corresponds to the $p$-polarized cavity input, whereas the Airy peak at $\Delta \approx-230 \mathrm{MHz}$ is related to the $s$-polarized local oscillator of our homodyne locking scheme.

frequency noise reductions are approximately equal when the cavity is locked. The PI controller is configured as follows:

$$
K_{\mathrm{PI}}(s)=\frac{0.1 s+316.5}{s}
$$
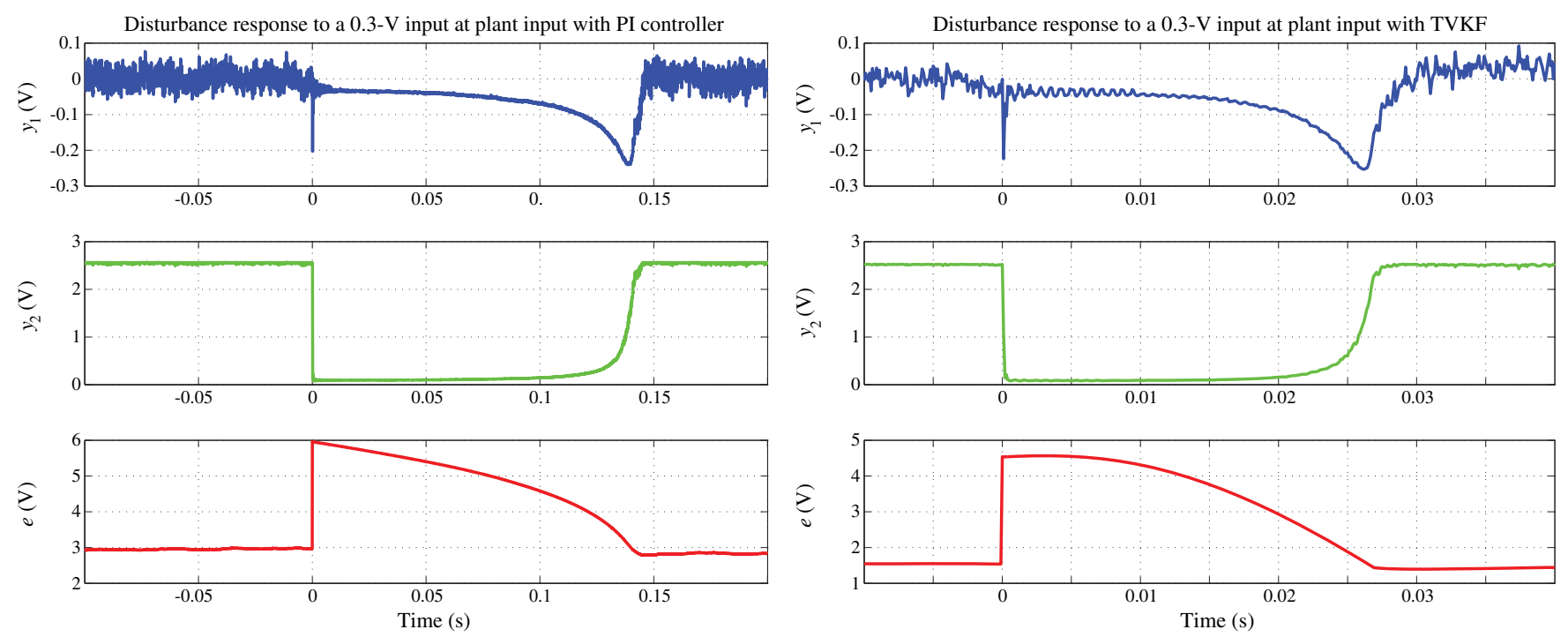

(a)

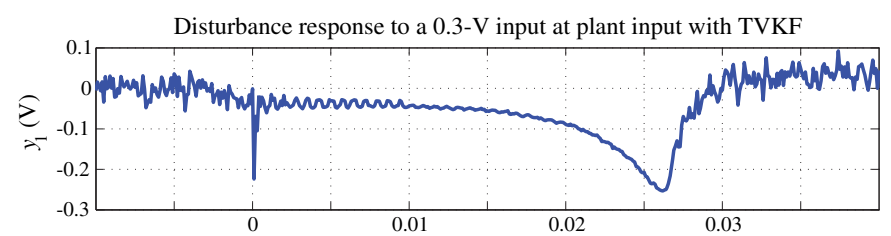

We first set up an experiment to demonstrate operation in the linear region, where conventional controllers can successfully relock the cavity. Then we highlight operation of our control scheme in the nonlinear region where the PI controller fails.

The quality and robustness of our locks are determined by injecting a disturbance in the form of a step function. For the locked case, the transmitted signal $y_{1}$ has its maximum value, and the error signal $y_{2}$ varies around zero, in contrast to the unlocked case where $y_{1}$ and $y_{2}$ are close to zero; see Fig. 6.

For the first test, we apply a step voltage to a piezoelectric actuator mounted to one of the cavity mirrors. The voltage step is $0.3 \mathrm{~V}$, which drives the detuning to the edge of the linear region. This disturbance is equivalent to a detuning of approximately $32 \mathrm{MHz}$ or $0.5 \kappa$. Figure 7 (a) shows the effect of the input disturbance on the system locked with the PI controller. The PI controller reacquires lock in 0.14 s. Figure 7(b) highlights the response of the proposed controller, which performs much faster, reacquiring lock in just $0.03 \mathrm{~s}$.

For the second test, we apply a step voltage of $0.4 \mathrm{~V}$ to the piezoelectric actuator. This drives the detuning into the nonlinear region and is equivalent to a detuning of approximately $43 \mathrm{MHz}$ or $0.66 \kappa$. As a result, the PI controller is unable to maintain cavity lock as shown in Fig. 8(a). On the other hand, our proposed control scheme does reacquire lock in approximately $35 \mathrm{~ms}$, as shown in Fig. 8(b). Figure 9 illustrates the transfer functions of the proposed controller at fixed instants of time after applying the step input at $t=0 \mathrm{~s}$. The controller transfer function varies in time since it depends on the operating point of

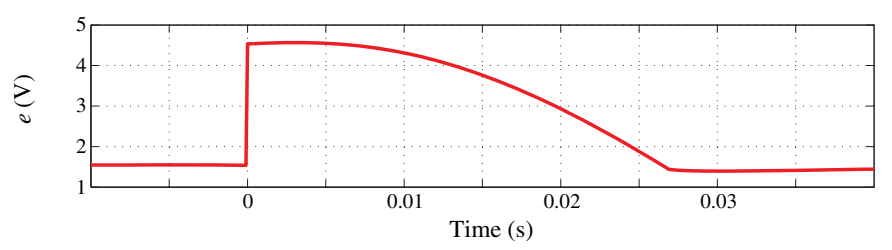

(b)

FIG. 7. Response of the optical system to an input disturbance step function of $0.3 \mathrm{~V}$, equivalent to a detuning $\Delta \approx 32 \mathrm{MHz} \approx 0.5 \kappa$, locked with a traditional PI controller (a) and the proposed controller (b). Our controller reacquires lock after $0.03 \mathrm{~s}$, which is superior to the PI controller reacquiring lock after $0.14 \mathrm{~s}$. 

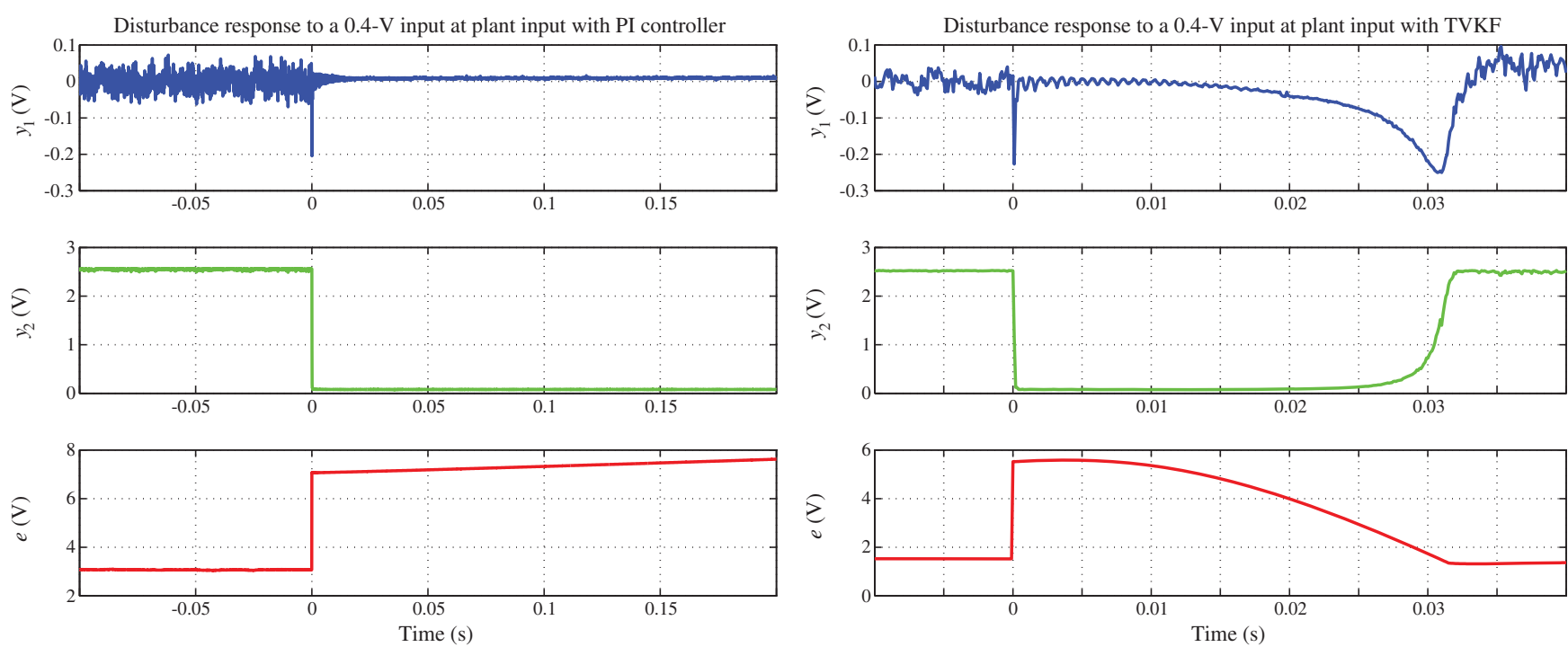

(a)

(b)

FIG. 8. Response of the optical system to an input disturbance step function of $0.4 \mathrm{~V}$, equivalent to a detuning $\Delta \approx 43 \mathrm{MHz} \approx 0.66 \kappa$ locked with the PI controller (a) and the proposed control scheme (b). Our control scheme reacquires lock after $35 \mathrm{~ms}$, whereas the PI controller fails to relock the cavity.

the system as well as the estimate of the detuning. The evolution of the controller transfer function is the reason why the time-varying Kalman filter is capable of operating in the nonlinear regime. Note that the plots for $t=-5 \mathrm{~ms}$ (system in lock before it is disturbed at $t=0 \mathrm{~s}$ ) and $t=35 \mathrm{~ms}$ (system reacquires lock) overlap and describe the optimal controller for the frequency-locked case.

When the detuning is very large, the measured output is essentially white noise. Since the augmented system includes an integrator, which integrates the output, the integrated state $q_{k}$ [see Eqs. (14) and (15)] is a Wiener process, which ensures that the controller converges; it will

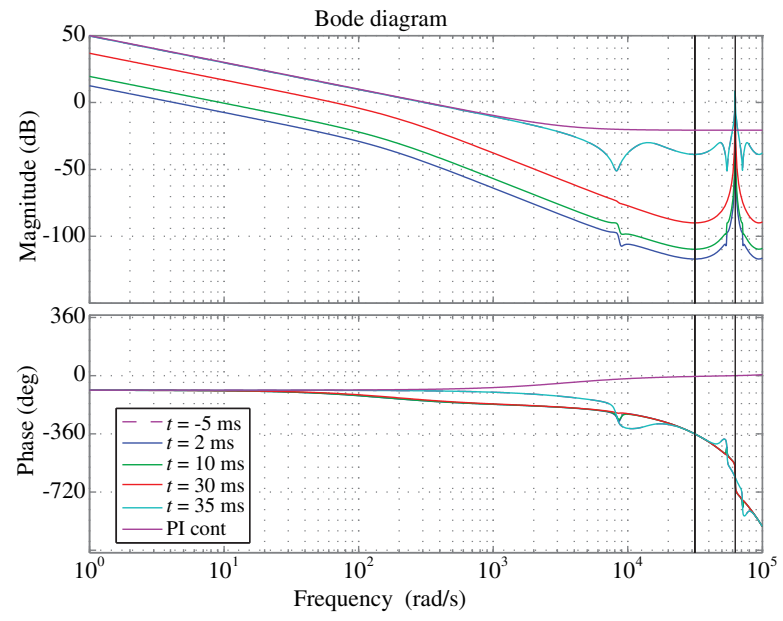

FIG. 9. The time-varying Kalman filter gives rise to a range of controllers at various instants of time, after applying a $0.4-\mathrm{V}$ step function disturbance at $t=0 \mathrm{~s}$. move in one direction until $y_{1}$ and $y_{2}$ become significant. Successful operation of the controller also confirms that we chose suitable bounds for the measurement noises [see Eq. (24)].

We successfully demonstrate the operation of the proposed control scheme, highlighting its ability to automatically reacquire cavity lock-without scanning the frequency - in an automated way. The PI controller, and all linear time-invariant controllers for that matter, will fail in the nonlinear region.

\section{CONCLUSION}

In this paper, we address the problem of automatically frequency locking an optical cavity from any given operating point. Our autolocking scheme successfully overcomes the inherent nonlinearity of the control problem which arises from the nonlinear error signal. We utilize techniques from modern control to simplify the nonlinear control problem to enable the application of well-known linear control techniques. Compared to a traditional PI controller, our controller reacquires lock in a significantly shorter time $(0.03 \mathrm{~s}$ as opposed to $0.14 \mathrm{~s})$ while in the linear region. We demonstrate that our autolocking scheme is capable of autonomously bringing the system back to resonance even in the presence of large disturbances, which cause the system to leave the linear region of operation, where linear time-invariant controllers fail. As our autolocking scheme significantly improves the robustness of the cavity lock (and, hence, the duty cycle), we believe this technique will be beneficial in a myriad of applications involving locked cavities. 


\section{ACKNOWLEDGMENTS}

This work is financially supported by the Deutsche Forschungsgemeinschaft through the Centre for Quantum Engineering and Space-Time Research (QUEST) and by the Australian Research Council. Furthermore, the authors thank the Quantum Control group from Hannover for the many fruitful discussions.

[1] L.-S. Ma and J. L. Hall, Optical heterodyne spectroscopy enhanced by an external optical cavity: Toward improved working standards, IEEE J. Quantum Electron. 26, 2006 (1990).

[2] B. A. Paldus, C. C. Harb, T. G. Spence, B. Wilke, J. Xie, J.S. Harris, and R. N. Zare, Cavity-locked ring-down spectroscopy, J. Appl. Phys. 83, 3991 (1998).

[3] S. L. Braunstein and P. Van Loock, Quantum information with continuous variables, Rev. Mod. Phys. 77, 513 (2005).

[4] J. S. Neergaard-Nielsen, B. M. Nielsen, C. Hettich, K. Mølmer, and E. S. Polzik, Generation of a Superposition of Odd Photon Number States for Quantum Information Networks, Phys. Rev. Lett. 97, 083604 (2006).

[5] N. Treps, U. Andersen, B. Buchler, P. K. Lam, A. Maître, H.-A. Bachor, and C. Fabre, Surpassing the Standard Quantum Limit for Optical Imaging Using Nonclassical Multimode Light, Phys. Rev. Lett. 88, 203601 (2002).

[6] C. Affeldt, K. Danzmann, K. L. Dooley, H. Grote, M. Hewitson, S. Hild, J. Hough, J. Leong, H. Lück, M. Prijatelj et al., Advanced techniques in GEO 600, Classical Quantum Gravity 31, 224002 (2014).

[7] J. Aasi, B. P. Abbott, R. Abbott, T. Abbott, M. R. Abernathy, K. Ackley, C. Adams, T. Adams, P. Addesso, R. X. Adhikari et al., Advanced LIGO, Classical Quantum Gravity 32, 115012 (2015).

[8] R. W. P. Drever, J. L. Hall, F. V. Kowalski, J. Hough, G. M. Ford, A. J. Munley, and H. Ward, Laser phase and frequency stabilization using an optical resonator, Appl. Phys. B 31, 97 (1983).
[9] T. W. Hänsch and B. Couillaud, Laser frequency stabilization by polarization spectroscopy of a reflecting reference cavity, Opt. Commun. 35, 441 (1980).

[10] M. Heurs, I. R. Petersen, M. R. James, and E. H. Huntington, Homodyne locking of a squeezer, Opt. Lett. 34, 2465 (2009).

[11] R. L. Barger, M.S. Sorem, and J. L. Hall, Frequency stabilization of a cw dye laser, Appl. Phys. Lett. 22, 573 (1973).

[12] G. Heinzel, Ph.D. thesis, Universität Hannover, 1999.

[13] S. Z. Sayed Hassen and I. R. Petersen, Frequency locking of an optical cavity using a time-varying Kalman filtering approach, IEEE Trans. Control Syst. Technol. 22, 1143 (2014).

[14] S. Z. Sayed Hassen, M. Heurs, E. H. Huntington, I. R. Petersen, and M. R. James, Frequency locking of an optical cavity using linear-quadratic Gaussian integral control, J. Phys. B 42, 175501 (2009).

[15] H.-A. Bachor and T. C. Ralph, A Guide to Experiments in Quantum Optics, 2nd ed. (Wiley, New York, 2004), Vol. 1.

[16] C. Gardiner and P. Zoller, Quantum Noise: A Handbook of Markovian and Non-Markovian Quantum Stochastic Methods with Applications to Quantum Optics (Springer Science \& Business Media, New York, 2004), Vol. 56.

[17] P. Van Overschee and B. L. R. De Moor, Subspace Identification for Linear Systems: Theory, Implementation, Applications (Kluwer Academic Publishers, Dordrecht, 1996), Vol. 3.

[18] P. Kokotovic, H. K. Khalil, and J. O'Reilly, Singular Perturbation Methods in Control: Analysis and Design (SIAM, 1999), Vol. 25.

[19] F.S. Acton, Numerical Methods that Usually Work (Mathematical Association of America Washington, DC, 1990).

[20] S. Goedecker, Remark on algorithms to find roots of polynomials, SIAM J. Sci. Comput. 15, 1059 (1994).

[21] R. G. Brown, Introduction to Random Signal Analysis and Kalman Filtering (Wiley, New York, 1983), Vol. 8.

[22] F. L. Lewis, Optimal Estimation: With an Introduction to Stochastic Control Theory (Wiley, New York, 1986), Vol. 1. 